\title{
Gravure printed PEDOT:PSS as anode for flexible ITO-free organic light emitting diodes
}

\author{
M. Montanino*, G. Sico, C. T. Prontera, A. De Girolamo Del Mauro, S. Aprano, \\ M. G. Maglione, C. Minarini
}

ENEA - Italian National Agency for New Technologies, Energy and Sustainable Economic Development, Portici Research Center, Piazzale E. Fermi 1, 80055 Portici (NA), Italy

Received 21 November 2016; accepted in revised form 25 January 2017

\begin{abstract}
Roll-to-roll gravure printing is considered as potential leading manufacturing technology for flexible, low cost and large area optoelectronics. However, solution processed multilayer organic electronics are still challenging to be produced, especially in the case of electrodes. In this work, the gravure printing technique was successfully employed to realize the highly conductive poly(3,4ethylenedioxythiophene):poly(styrene sulfonate) (PEDOT:PSS) polymeric anode and tested for the first time in flexible ITO-free (Indium Thin Oxide) organic light emitting diodes (OLEDs). The device performances were found to be similar to those of a reference device containing a spin-coated polymeric anode. A gravure printed dimethyl sulfoxide (DMSO) post-treatment was successfully tried to improve the printed anode characteristics. The obtained results show the way for future development for processing flexible ITO-free devices using the most attractive printing technology for roll-to-roll large area manufacturing.
\end{abstract}

Keywords: industrial applications, PEDOT:PSS, ITO-free, flexible organic light emitting diode, gravure printing

\section{Introduction}

Organic light emitting diodes (OLEDs) are an emerging technology for displays and lighting applications which are gaining more and more attention because of their simple structure, low weight and low cost processing potentials [1]. In particular, polymer light emitting diodes (PLEDs) are very interesting for the possibility to be fabricated on flexible substrates by solution processes compatible with roll-to-roll printing and coating techniques $[2,3]$. The decrease of the fabrication process costs is in fact one of the most important topics related to these devices. Currently, the widely used technique to realize the OLEDs layers on lab scale is the spin coating. However, such technique is not suitable for the industrial production due to its low throughput, large waste of materials and incompatibility with large areas, while roll-to-roll printing and coating processes appear very promising in coupling the low cost and large area production of flexible devices. Among the printing techniques, the gravure is the best candidate for such propose since it is very well-established and widely used in graphic and packaging industry offering many advantages such as high speed, high quality and high versatility; moreover, it is especially suited for flexible substrates and for continuous multilayer production of large area arbitrary shape $[4,5]$. The gravure printing is a multi-step process: a micro-engraved cylinder, continuously filled of low viscosity ink (inking), whose excess is removed by a blade (doctoring), transfers the ink by means of the pressure of a rubber cylinder (transfer) directly onto the substrate where successively the ink spreads (spreading). Despite its many advantages, to date, the use of the gravure

${ }^{*}$ Corresponding author, e-mail: maria.montanino@enea.it

(C) BME-PT 
printing for the production of the optoelectronics is very limited mainly due to the serious difficulties in the formulation of low viscosity inks able to form layers with suitable characteristics. The few manuscripts on the employment of the gravure printing in the production of OLEDs are typically confined to the deposition of the polymeric Hole Injection Layer (HIL) and/or of the emitting layer [3, 4, 6-8]. Up to date, the most investigated OLED devices are based on the rigid glass substrate and on the brittle and expensive Indium Tin Oxide (ITO) as transparent electrode [4], while the research is focusing on flexible devices and on the substitution of ITO with other conductive materials. Among the polymeric layer able to act as electrode, the highly conductive (HC) poly $(3,4$ ethylenedioxythiophene):poly(styrene sulfonate) (PEDOT:PSS), obtained by the addition of dimethyl sulfoxide (DMSO), is one of the most promising candidates [9].

In an our previous work, gravure printing of $\mathrm{HC}$ PEDOT:PSS onto flexible polyethylene naphthalate (PEN) substrate was investigated with the aim to obtain a layer suitable to be used as transparent anode in flexible electronic [10]. Such layer was obtained thanks to an easy approach able to improve it in terms of morphological and electrical characteristics, and is based on the multilayer printing of inks having decreasing concentrations. In this work, for the first time, such gravure printed HC PEDOT:PSS is proved as anode for flexible ITO-free PLED, promoting the possibility to employ the use of the gravure printing technique in the OLEDs preparation. The investigated PLEDs were also composed of a commercial spin coated polymer as emitting layer and evaporated $\mathrm{Ca} / \mathrm{Ag}$ as cathode. For comparison, HC PEDOT:PSS deposited by spin coating was proved in OLEDs having the same structure. A further improvement of the gravure printed PEDOT:PSS layer through a solvent printing post-treatment was also reported and tested in similar devices. The produced layers were characterized in terms of optical, morphological and electrical proprieties and then tested in the devices.

\section{Experimental section}

All the inks were developed and gravure printed or spin coated in air at room temperature on polyethylene naphthalate (PEN) substrate (Teonex ${ }^{\circledR}$ Q65FA, DuPont Teijin Films, Chester VA, USA) provided in A4 sheet, having a thickness of $125 \mu \mathrm{m}$.
The inks to realize the anode were prepared using a commercially available aqueous PEDOT:PSS dispersion (Clevious PH 1000, Heraeus Clevios GmbH, Leverkusen, Germany). The dimethylsulfoxide (DMSO) was added ( $5 \%$ by volume) in order to enhance its conductivity, as also suggested by the supplier [11]. This pristine solution, suitable for spin-coating deposition, was diluted by 1,2-isopropyl alcohol (IPA) in different weight ratios to decrease the viscosity and the surface tension, obtaining inks gravure printable without the use of any substrate pre-treatment [10]. The reference anodic layers were obtained spin coating the pristine solution at $800 \mathrm{rpm}$ for $60 \mathrm{sec}$ on PEN treated with Ultra Violet (UV)-ozone to improve the wettability of ink. The films were subsequently annealed at $140^{\circ} \mathrm{C}$ for all night.

The employed gravure was a lab-scale printer (G1-5, IGT, Amsterdam, The Netherlands) equipped with a cylinder having line density of 40 lines/cm, stylus angle of $120^{\circ}$, cell depth of $72 \mu \mathrm{m}$ and screen angle of $53^{\circ}$. The diluted inks were gravure printed using a printing force of $1000 \mathrm{~N}$ at a speed of $60 \mathrm{~m} / \mathrm{min}$. The multilayer structure was obtained by drying each layer at $140^{\circ} \mathrm{C}$ for $5 \mathrm{~min}$ before printing the next one. The optimized polymeric anode was found to be a three printed layers following a decreasing profile as reported elsewhere [10]. The drying time and temperature conditions were chosen to be industrially sustainable: shorter drying period results in a worst printing quality of the successive layer and the temperature was kept the same used for the final thermal annealing overnight. In order to further improve the conductivity of the printed layer, in some cases, a post-treatment was performed by gravure printing DMSO solvent on the printed anode, before the final annealing, using a printing force of $500 \mathrm{~N}$ at a speed of $12 \mathrm{~m} / \mathrm{min}$; the layer was then annealed at the same process temperature after a slow solvent evaporation $\left(70^{\circ} \mathrm{C}\right.$ for $\left.5 \mathrm{~min}\right)$. Such conditions were found to be the best among the tested conditions for this treatment. The thickness and surface roughness of the coated/ printed layers were investigated by interferometrybased optical profilometer (Talysurf CCI HD, Taylor Hobson, Leicester, UK). The root mean square surface roughness was obtained according to the ISO 25178 standard; the reported values are the average results of the measurements made.

The electrical conductivity of the anodes was evaluated by sheets resistance measurements performed by 
a four points probe instrument (Resistest RT $8 \mathrm{~A}$ coupled with Resistage RG 8, Napson, Tokyo, Japan) applying a current of $1 \mu \mathrm{A}$.

The produced films were also characterized by performing UV-visible transmission measurements (Lambda 900, Perkin Elmer, Waltham MA, USA) and by scanning electron microscopy (1530, LEO Elektronenmikroskopie GmbH, Oberkochen, Germany), respectively.

Concerning the devices preparation, the active layer was obtained through spin coating a solution containing $10 \mathrm{mg} / \mathrm{mL}$ of a commercial white emitting polymer (Livilux SPW 111, Merck, Darmstadt, Germany) in toluene at $3000 \mathrm{rpm}$ for all the prepared devices. The active layers had a thickness of $\approx 100 \mathrm{~nm}$ as measured by profilometer. The $\mathrm{Ca} / \mathrm{Ag}$ cathode $(20$ and $80 \mathrm{~nm}$ respectively) was deposited by thermal evaporation in ultra-high vacuum (10-7 mbar) using a shadow mask to define the active area of the device $\left(0.07 \mathrm{~cm}^{2}\right)$.

Electrical and optical characteristics of the devices were measured using respectively a sourcemeter (Keithley 2400, Tektronix, Beaverton OR, USA) and spectroradiometer (OL770, Gooch\&Housego, Ilminster Somerset, UK) coupled with an integrating sphere. Several devices (ten per type) were tested to verify the reproducibility of the results. The reported data are related to the best results obtained in the device for the different investigated anodes.

\section{Results and discussion}

In a previous paper, we demonstrated the possibility to produce by gravure printing HC PEDOT:PSS layer suitable as transparent anode for electronic devices thanks to a multilayer approach, using IPA as process solvent; in fact, a single layer produced by the highest concentrated printable ink was not suitable as anode even when printed using high grammage cylinder [10]. Therefore, overlap of more printed layers was adopted as a possible solution. The multilayer approach allowed to increase the conductivity of the $\mathrm{HC}$ PEDOT:PSS thanks to the decrease of the sheet resistance with increasing the number of the overlapped layers, keeping an high transparency. The best lay down of the consecutive printed layers was obtained overlapping inks at decreasing concentration: the increase in the solvent content of the consecutive printed layers allowed to achieve values of conductivity higher than the multilayer printed at the same ink concentration. In particular, just three layers successively printed with decreasing concentration profile of $\mathrm{HC}$ PEDOT:PSS permitted to reach characteristics suitable for electrode applications, thanks to morphological improvements related to the better spreading of the material in the successive printed layers and to the smoothing effect on the previous printed layers. Gravure printing has a complex nature that involves several physical phenomena and can be considered composed of some sub-processes (inking, doctoring, transfer and spreading), each of which has its ideal operating regime. The final printing quality is determined by how these sub-processes are approaching the ideality [12] and this is very important in the case of the optoelectronics and in particular of the OLEDs: any possible defects can generate non-uniform emission or, in the worst case, short circuits due to the pinholes $[13,14]$. Therefore, the printed layers should be defect-free, uniform and homogeneous with very controlled thicknesses $[6-8,15]$; very low roughness is also a crucial feature since the morphology of the previous printed layers can influence the properties of the successive layers [14]. In order to further improve the quality of the produced layer with the aim to prove it as anode into an OLED, increasing the printing speed was here tried without changing ink (viscosity, solvent) and other process parameters (cells geometry, nip pressure), looking for the optimal conditions of the ink transfer step [12,15-17].

In Table 1 the characteristics of the printed anodes are showed and compared with the ones of the anode obtained by spin coating. In Figure 1 the transparency of the layers is proved through the reported UV transmittance measurements. The properties of the layers are very similar, despite the great differences between the fabrication techniques: the gravure printing is in fact discontinuous being applied through a

Table 1. Electrical and morphological characteristics of the spin coated and the gravure printed HC PEDOT deposited on PEN substrate; the conductivity values are calculated by the average values of the thickness and the sheet resistances

\begin{tabular}{|c|c|c|c|c|}
\hline Anode preparation technique & $\begin{array}{c}\text { Thickness } \\
\text { [nm] }\end{array}$ & $\begin{array}{c}\text { Sheet resistance } \\
{[\Omega / s q]}\end{array}$ & $\begin{array}{c}\text { Conductivity } \\
{[\mathrm{S} / \mathrm{cm}]}\end{array}$ & $\begin{array}{c}\text { Surface roughness } \\
{[\mathrm{nm}]}\end{array}$ \\
\hline Spin coating & $162 \pm 8$ & $137 \pm 8$ & 451 & $11 \pm 1$ \\
\hline Multilayer gravure printing & $157 \pm 10$ & $146 \pm 10$ & 436 & $21 \pm 3$ \\
\hline Multilayer gravure printing + solvent post treatment & $151 \pm 6$ & $125 \pm 5$ & 530 & $18 \pm 2$ \\
\hline
\end{tabular}




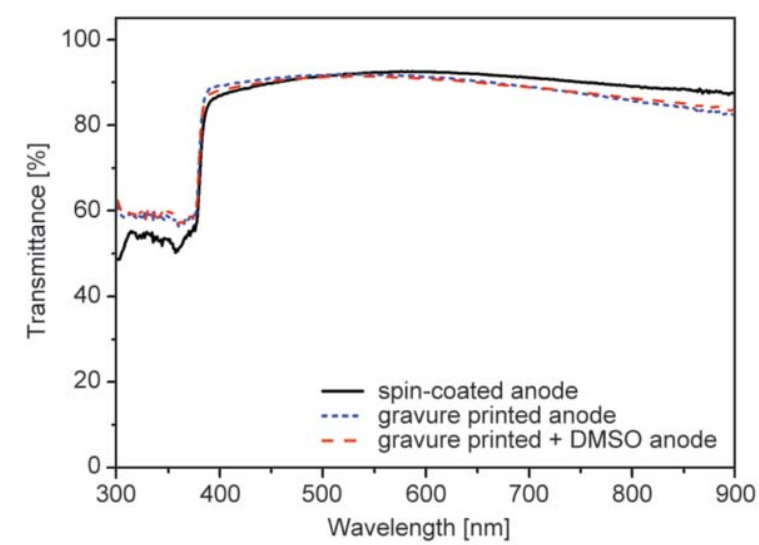

Figure 1. UV transmittance measurements of the spin coated and gravure printed layers on the PEN substrate

multi-step process and it is intrinsically less uniform than the spin coating involving the coalescence of the transferred inks droplets before solvent evaporation $[3,13]$. This demonstrated the possibility to obtain layers by gravure printing of comparable quality to the widely used coating technique, promoting the use of the printing technique in all the applications for which the spin coating is used with the advantage to be easily industrially scalable. As expected, the roughness of the gravure printed anodes is higher than the one of the spin coated layers mainly due to the overlapping process.

The characteristics of the gravure printed anode were then further improved thanks to a solvent printing post-treatment. As proposed in literature, treating the HC PEDOT:PSS layer with DMSO leads to a removal of the insulating PSS components promoting the connection among the conducting PEDOT grains and thus improving the overall conductivity of the layer [18-20]. Here, for the first time, such DMSO post-treatment was tried through gravure printing in the perspective of a process completely made by such technique. As expected, the post-treatment was found to increase the conductivity (plus 20\%) of the printed layer; the effect of the solvent post-treatment causes also a slightly smoothing effect on the surface roughness of the pre-printed layer and an increase in its uniformity (see Table 1). SEM analysis confirms an increase of the quantity of the PEDOT grains and a higher uniformity of the grain size in the printed sample treated with DMSO (Figure 2c) with respect to the untreated one (Figure 2b). In fact, the gravure printed sample (Figure $2 b$ ) shows an inhomogeneous distribution of the domains on its surface also very different in size (below 1 up to $5 \mu \mathrm{m}$ ), while the gravure printed+DMSO anode shows an uniformity of the

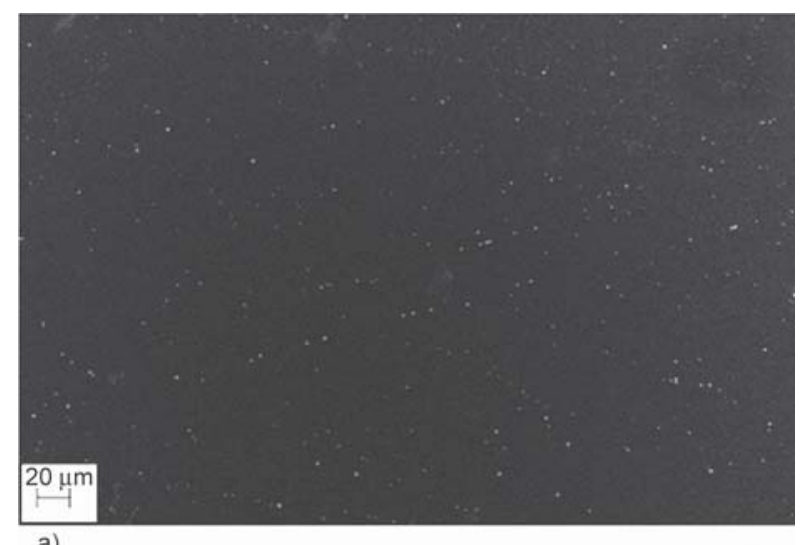

a)

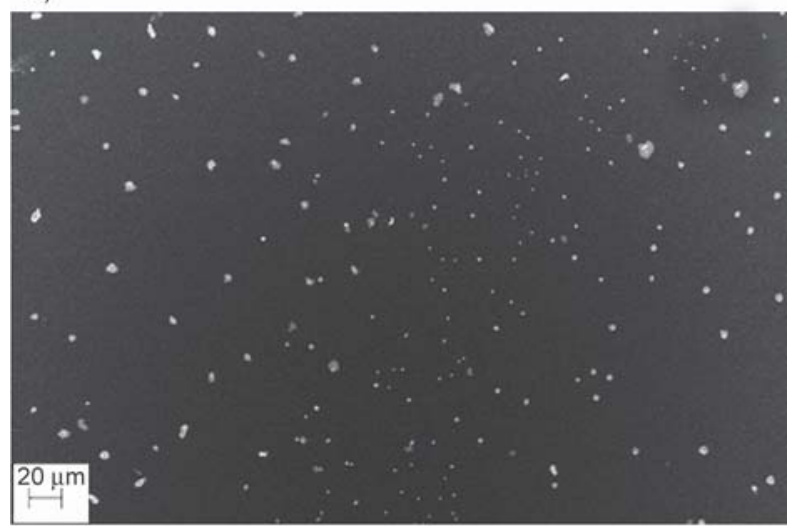

b)

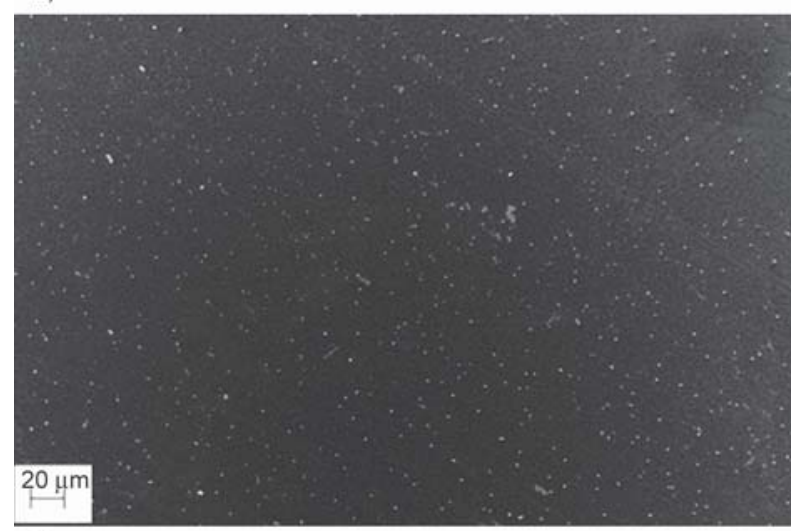

c)

Figure 2. SEM images of the spin coated (a), gravure printed (b) and gravure printed with DMSO post treatment (c) HC PEDOT:PSS

PEDOT domains, in terms of size $(\leq 1 \mu \mathrm{m})$ and distribution, similar to the spin coated sample (Figure 2a) but in a higher density.

The produced anodes were tested in OLEDs having the following structure: PEN/HC PEDOT:PSS/Emitting polymer/Ca/Ag. A levelling effect of the spin coated emitting polymer on the roughness of the different anodes deposited on PEN substrate was found (see Table 2$)$. The current density vs voltage $(J-V)$ curves of the devices are reported in Figure 3, while the luminance vs voltage characteristics are reported in Figure 4. As expected, the performances of the 
Table 2. Surface roughness of the emitting polymer layer spin coated onto the spin coated and the gravure printed HC PEDOT:PSS anodes deposited on PEN substrate

\begin{tabular}{|l|c|}
\hline \multicolumn{1}{|c|}{$\begin{array}{c}\text { Preparation technique } \\
\text { (HC PEDOT:PSS/emitting polymer) }\end{array}$} & $\begin{array}{c}\text { Surface roughness } \\
\text { [nm] }\end{array}$ \\
\hline Spin coating/spin coating & $12 \pm 2$ \\
\hline Multilayer gravure printing/spin coating & $18 \pm 2$ \\
\hline $\begin{array}{l}\text { Multilayer gravure printing + } \\
\text { solvent post treatment/spin coating }\end{array}$ & $14 \pm 1$ \\
\hline
\end{tabular}

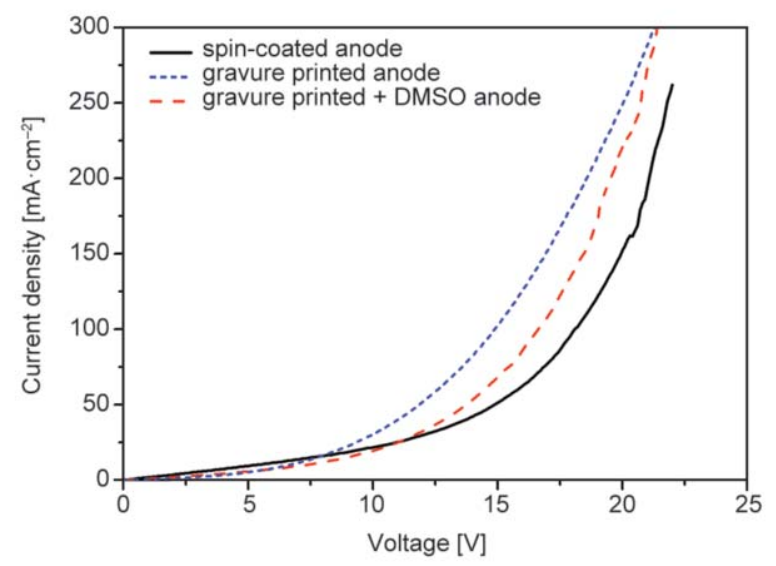

Figure 3. $J-V$ curves of the fabricated OLEDs containing anodes obtained by spin coating, by gravure printing and by gravure printing plus DMSO post treatment

OLEDs containing the gravure printed and the spin coated anodes are similar since the characteristics of their electrodes are similar.

Considering the obtained luminance values, it does not appear possible to discriminate significant differences in the behavior of the anodes in the devices. Anyway, the slightly observed differences can be related to the differences in the anodes and devices

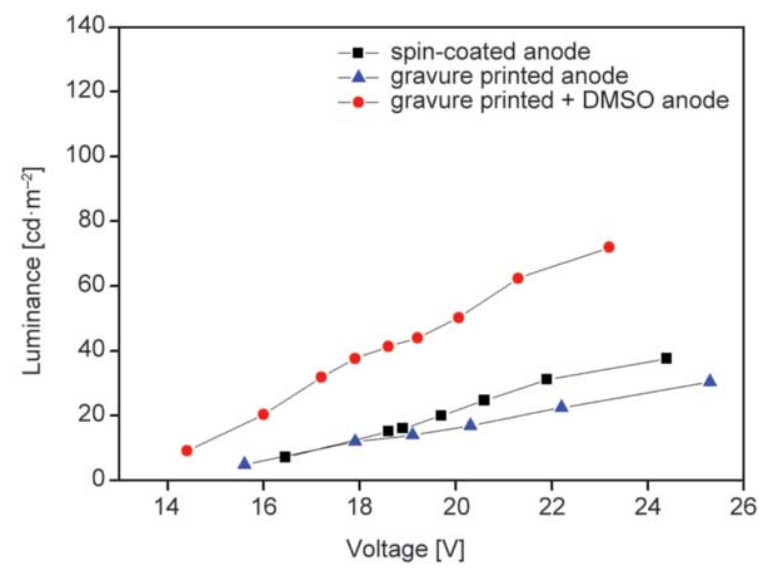

Figure 4. Luminance vs Voltage characteristics of the fabricated OLEDs containing anodes obtained by spin coating, by gravure printing and by gravure printing plus DMSO post-treatment manufacturing. However, since the enhancement of the post-treatment on the printed layer, this study, focused on the process feasibility, indicates the direction to follow to further improve the layer and to optimize the process.

In conclusion, the present study proves that multilayer gravure printed HC PEDOT:PSS, realized with increasingly dilution profile stacking, can be effectively used as anode for OLEDs fabrication, expanding the possibilities for future developments of optoelectronic devices, partly or entirely, industrially produced by gravure printing. The obtained performances, relatively poor if compared to typical ITO and glass based OLEDs, are therefore largely rewarded by the demonstrated opportunity for processing flexible ITO-free devices using the most attractive printing technology for roll-to-roll large area manufacturing.

\section{Conclusions}

For the first time gravure printed HC PEDOT:PSS anode was used to realize flexible ITO-free OLEDs. In this work, we demonstrated that the gravure printing is a viable technique to realize such devices which require the highest uniformity of their constituting layers, reaching the same quality of the layers obtained by spin coating lab-scale confined process even in the most complicate case of the polymeric electrode. Thanks to the possibility to employ the only high speed and high resolution printing technique for the partial or complete production of large area, arbitrary shape OLEDs and, in general, flexible organic electronic devices, this work offers the possibility to strongly decrease the production costs and to easy industrialize the process itself. Finally, a solvent posttreatment for improving the printed electrode quality was successfully performed by gravure, further showing the way for all in-line one-step printing process.

\section{Acknowledgements}

This work has been supported by the Italian Ministry of Education, University and Research (MIUR) through the National Project entitled RELIGHT (PN: PON02 00556 3306937).

\section{References}

[1] Weiss O. J., Krause R., Paetzold R.: Organic thin film devices for displays and lighting. in 'Advances in solid state physics’ (ed.: Haug R.), Springer, Berlin, Vol 46, 321-332 (2008).

https://doi.org/10.1007/978-3-540-38235-5_24 
[2] Harding M. J., Poplavskyy D., Choong V-E., Campbell A. J., So F.: Effects of solution-processed polymer interlayers on hole injection and device performance of polymer light-emitting diodes. Organic Electronics, 9, 183-190 (2008).

https://doi.org/10.1016/j.orgel.2007.09.003

[3] Chung D-Y., Huang J., Bradley D. D. C., Campbell A. J.: High performance, flexible polymer light-emitting diodes (PLEDs) with gravure contact printed hole injection and light emitting layers. Organic Electronics, 11, 1088-1095 (2010).

https://doi.org/10.1016/j.orgel.2010.03.010

[4] Søndergaard R. R., Hösel M., Krebs F. C.: Roll-to-roll fabrication of large area functional organic materials. Journal of Polymer Science Part B: Polymer Physics, 51, 16-34 (2013).

https://doi.org/10.1002/polb.23192

[5] Kopola P., Aernouts T., Guillerez S., Jin H., Tuomikoski M., Maaninen A., Hast J.: High efficient plastic solar cells fabricated with a high-throughput gravure printing method. Solar Energy Materials and Solar Cells, 94, 1673-1680 (2010).

https://doi.org/10.1016/j.solmat.2010.05.027

[6] Lee H., Kim A., Cho S. M., Chae H.: Characterization of thermal annealing of gravure printed PVK/Ir(ppy)3 organic light emitting layers. Current Applied Physics, 10, 143-146 (2010). https://doi.org/10.1016/j.cap.2010.08.029

[7] Tekoglu S., Hernandez-Sosa G., Kluge E., Lemmer U., Mechau N.: Gravure printed flexible small-molecule organic light emitting diodes. Organic Electronics, 14, 3493-3499 (2013).

https://doi.org/10.1016/j.orgel.2013.09.027

[8] Hernandez-Sosa G., Tekoglu S., Stolz S., Eckstein R., Teusch C., Trapp J., Lemmer U., Hamburger M., Mechau N.: The compromises of printing organic electronics: A case study of gravure-printed light-emitting electrochemical cells. Advanced Materials, 26, 3235-3240 (2014). https://doi.org/10.1002/adma.201305541

[9] Wang G-F., Tao X-M., Xin J. H., Fei B.: Modification of conductive polymer for polymeric anodes of flexible organic light-emitting diodes. Nanoscale Research Letters, 4, 613-617 (2009).

https://doi.org/10.1007/s11671-009-9288-8

[10] Sico G., Montanino M., De Girolamo Del Mauro A., Imparato A., Nobile G., Minarini C.: Effects of the ink concentration on multi-layer gravure-printed PEDOT: PSS. Organic Electronics, 28, 257-262 (2016). https://doi.org/10.1016/j.orgel.2015.10.031

[11] De Girolamo Del Mauro A., Nenna G., Villani F., Minarini C.: Study of the effect of the doped poly $(3,4-e t h-$ ylenedioxythiophene):poly(styrene sulfonate) polymeric anode on the organic light-emitting diode performances. Thin Solid Films, 520, 5386-5391 (2012).

https://doi.org/10.1016/j.tsf.2012.03.128
[12] Grau G., Cen J., Kang H., Kitsomboonloha R., Scheideler W. J., Subramanian V.: Gravure-printed electronics: Recent progress in tooling development, understanding of printing physics, and realization of printed devices. Flexible and Printed Electronics, 1, 1-23 (2016). https://doi.org/10.1088/2058-8585/1/2/023002

[13] Kopola P., Tuomikoski M., Suhonen R., Maaninen A.: Gravure printed organic light emitting diodes for lighting applications. Thin Solid Films, 517, 5757-5762 (2009).

https://doi.org/10.1016/j.tsf.2009.03.209

[14] Michels J. J., de Winter S. H. P. M., Symonds L. H. G.: Process optimization of gravure printed light-emitting polymer layers by a neural network approach. Organic Electronics, 10, 1495-1504 (2009). https://doi.org/10.1016/j.orgel.2009.08.015

[15] Noh J., Yeom D., Lim C., Cha H., Han J., Kim J., Park Y., Subramanian V., Cho G.: Scalability of roll-to-roll gravure-printed electrodes on plastic foils. IEEE Transactions on Electronics Packaging Manufacturing, 33, 275-283 (2010). https://doi.org/10.1109/TEPM.2010.2057512

[16] Nguyen H. A. D., Lee J., Kim C. H., Shin K-H., Lee D.: An approach for controlling printed line-width in high resolution roll-to-roll gravure printing. Journal of Micromechanics and Microengineering, 23, 095010/1095010/10 (2013). https://doi.org/10.1088/0960-1317/23/9/095010

[17] Sankaran A. K., Rothstein J. P.: Effect of viscoelasticity on liquid transfer during gravure printing. Journal of NonNewtonian Fluid Mechanics, 175-176, 64-75 (2012). https://doi.org/10.1016/j.jnnfm.2012.03.011

[18] Yeo J-S., Yun J-M., Kim D-Y., Kim S-S., Na S-I.: Successive solvent-treated PEDOT:PSS electrodes for flexible ITO-free organic photovoltaics. Solar Energy Materials and Solar Cells, 114, 104-109 (2013).

https://doi.org/10.1016/j.solmat.2013.02.031

[19] Sun K., Zhang S., Li P., Xia Y., Zhang X., Du D., Isikgor F. H., Ouyang J.: Review on application of PEDOTs and PEDOT:PSS in energy conversion and storage devices. Journal of Materials Science: Materials in Electronics, 26, 4438-4462 (2015). https://doi.org/10.1007/s10854-015-2895-5

[20] Wang M., Zhou M., Zhu L., Li Q., Jiang C.: Enhanced polymer solar cells efficiency by surface coating of the PEDOT: PSS with polar solvent. Solar Energy, 129, 175-183 (2016). https://doi.org/10.1016/j.solener.2016.02.003 